\title{
Safety of Positive Pressure Extubation Technique
}

\author{
Mauro F Andreu, María E Dotta, Marco G Bezzi, Silvina Borello, Gimena P Cardoso, \\ Paula C Dib, Silvina L García Schustereder, Alejandra M Galloli, Daniela R Castro, \\ Victoria L Di Giorgio, Federico J Villalba, Matías N Bertozzi, Juan M Carballo, María C Martín, \\ Carla C Brovia, María C Pita, María P Pedace, María F De Benedetto, Julieta Delli Carpini, \\ Patricio Aguirre, and Gisela Montero
}

\begin{abstract}
BACKGROUND: Laboratory studies suggest applying positive pressure without endotracheal suction during cuff deflation and extubation. Although some studies reported better physiological outcomes (e.g. arterial blood gases) with this technique, the safety of positive pressure extubation technique has not been well studied. The aim of this study was to determine the safety of the positive-pressure extubation technique compared with the traditional extubation technique in terms of incidence of complications. METHODS: Adult subjects who were critically ill and on invasive mechanical ventilation who met extubation criteria were included. The subjects were randomly assigned to positive-pressure extubation $(n=120)$ or to traditional extubation $(n=120)$. Sequential tests for noninferiority and, when appropriate, for superiority were performed. Positive pressure was considered noninferior if the upper limit of the CI for the absolute risk difference did not exceed a threshold of $15 \%$ in favor of the traditional group, both in per protocol and intentionto-treat analyses. A $P$ value of $<.05$ was considered significant. RESULTS: A total of 236 subjects were included in the primary analysis (per protocol) (119 in the positive-pressure group and 117 in the traditional group). The incidence of overall major and minor complications, pneumonia, extubation failure, and reintubation was lower in the positive-pressure group than in the traditional group, with statistical significance for noninferiority both in the per protocol $(P<.001)$ and intention-to-treat $(P<.001)$ analyses. The lower incidence of major complications found in the positive-pressure group reached statistical significance for the superiority comparison, both in per protocol $(P=.03)$ and intention-to-treat $(P=.049)$ analyses. No statistically significant differences were found in the superiority comparison for overall complications, minor complications, pneumonia, extubation failure, and reintubation. CONCLUSIONS: Positive pressure was safe and noninferior to traditional extubation methods. Furthermore, positive pressure has shown to be superior in terms of a lower incidence of major complications. (ClinicalTrials.gov registration NCT03174509.) Key words: airway extubation; extubation methods; extubation complications; positive pressure; positivepressure extubation; tracheal extubation; positive-pressure ventilation. [Respir Care 2019;64(8):899-907. (C) 2019 Daedalus Enterprises]
\end{abstract}

\section{Introduction}

Extubation is the removal of an endotracheal tube (ETT) when it is no longer needed. ${ }^{1}$ This procedure may be as-

Mr Andreu and Mr Bertozzi are affiliated with the Department of Health Sciences, Physical Therapy, Universidad de la Matanza, San Justo, Buenos Aires, Argentina. Mr Andreu, Ms Dotta, Mr Bezzi, Ms Borello, Ms Cardoso, Ms Dib, Ms García Schustereder, Ms Galloli, Ms Castro, Ms Di Giorgio, Mr Villalba, Mr Bertozzi, Mr Carballo, Ms Martín, Ms Brovia, sociated with complications, including, for example, desaturation, stridor, bronchospasm, and severe cough., ${ }^{2,3}$ Complication rates reported in the literature range from $6.6 \%$ to $100 \% .^{4-6}$ In some cases, these complications may lead to extubation failure. ${ }^{7}$ Two extubation methods are Ms Pita, Ms Pedace, Ms De Benedetto, Ms Delli Carpini, Mr Aguirre,
and Ms Montero are affiliated with the Division of Respiratory Care
and Physical Therapy, Hospital Donación Francisco Santojanni, 
reported in the literature. ${ }^{8}$ Traditional extubation consists of introducing a suction catheter into the ETT and the trachea, and then deflating the cuff and removing the ETT with continuous suctioning during the whole procedure. The positive-pressure technique involves applying a pressure level during cuff deflation and extubation, thus, the air flow passing between the ETT and the larynx expels pooled subglottic secretions toward the oropharynx so that they can be suctioned once they have reached the oral cavity. ${ }^{8,9}$ Both approaches seek to minimize leakage of this oropharyngeal content into the distal airway. However, some investigators have argued that, with the traditional method, negative suction pressure could favor aspiration rather than prevent it. 9,10

Two laboratory studies showed that applying positive pressure without endotracheal suction during cuff deflation and extubation results in less leak volume. ${ }^{911}$ Although some studies reported physiological outcomes with this technique, its safety has not been well studied.6,12 Before assessing the superiority and applicability of extubation with positive pressure in daily clinical practice, we consider it essential to ensure the safety of this technique. Thus, our study objective was to determine the safety of the positive-pressure extubation compared with the traditional extubation in terms of the incidence of overall complications. We hypothesized that positive pressure would be noninferior to traditional extubation.

\section{Methods}

\section{Design}

A noninferiority clinical trial was performed. From January 2017 to March 2018, subjects in the ICU, the emergency department, and the coronary unit at Hospital Donación Francisco Santojanni were consecutively included in the study. It was approved by the hospital ethics committee (HGADFS14012016-01) and is registered on ClinicalTrials.gov. This study was reported by using the Consolidated Standards of Reporting Trials statement guidelines for noninferiority studies. ${ }^{13,14}$

\footnotetext{
Buenos Aires, Argentina. Mr Andreu is affiliated with the Division of Respiratory Care, Intensive Care Unit, Clínica Olivos, Swiss Medical Group, Buenos Aires, Argentina.
}

The authors have disclosed no conflicts of interest.

Correspondence: Mauro Andreu PT, Department of Health Sciences, Division of Physical Therapy, Universidad de la Matanza, Florencio Varela 1903, B1754, San Justo, Buenos Aires, Argentina. Email: maufede@hotmail.com.

DOI: $10.4187 /$ respcare. 06541

\section{QUICK LOOK}

\section{Current knowledge}

Orotracheal extubation may be associated with different complications. Two extubation methods are described in the literature: the traditional method and the positive-pressure method. Laboratory studies suggest applying positive pressure without endotracheal suction during cuff deflation and extubation. Although some studies reported better physiological outcomes with this technique, its safety has not been well studied.

\section{What this paper contributes to our knowledge}

Orotracheal extubation with positive pressure and without endotracheal suction was a safe technique and could be better than traditional extubation. The positive pressure extubation group was statistically safer overall, and had lower rates of major complications.

\section{Eligibility Criteria}

The study included all subjects ages $>18$ y who required invasive mechanical ventilation through an ETT, who had successfully completed a T-tube spontaneous breathing trial that lasted $30 \mathrm{~min},{ }^{15}$ and who met the following extubation criteria: adequate level of consciousness (Glasgow Coma Score $\geq 8$ ), ${ }^{16}$ a small amount of secretions (defined as suction requirement every $\geq 2 \mathrm{~h}$ within $6 \mathrm{~h}$ before extubation), ${ }^{17}$ and effective cough (a score of 3 or 4 on the scale described by Thille et al ${ }^{18}$ ). Written informed consent was obtained from a relative or legal representative. Patients with a history of upper airway injury or surgery, who had previously been extubated or tracheostomized, or who had received noninvasive ventilation (NIV) as a weaning method were excluded.

\section{Interventions}

Traditional extubation was performed by 2 operators. Without reconnection to the ventilator, the closed suction system catheter was introduced by one of the operators into the ETT and suctioning was initiated. The cuff was immediately deflated by the other operator, and the ETT was removed with continuous endotracheal suction during the whole procedure by the first operator. ${ }^{8}$ Positive-pressure extubation was performed by only one operator. Ventilator parameters were set to pressure support ventilation mode, with an inspiratory pressure of $15 \mathrm{~cm} \mathrm{H}_{2} \mathrm{O}$ and PEEP of $10 \mathrm{~cm} \mathrm{H}_{2} \mathrm{O}$. Then, the cuff was deflated, and the ETT was removed without endotracheal suction. Once the ETT was removed, a suction catheter was introduced 
through the mouth to suction secretions drawn to the oropharynx by the air flow from the ventilator passing between the ETT and the larynx..$^{8,11}$ Regardless of the extubation technique used, the subjects received supplemental oxygen at the same flow level used for the spontaneous breathing trial. In case of desaturation, the flow was regulated to maintain an oxygen saturation of $>90 \%$. Respiratory therapists who completed training in both extubation techniques before the beginning of the study performed every extubation procedure.

\section{Extubation Procedure}

Once the subjects had completed the spontaneous breathing trial and met extubation criteria, they entered the study and were assigned to an intervention group. A pre-extubation procedure was performed by operator A (evaluator). This procedure consisted of discontinuation of enteral nutrition, adjusting the head of the bed to 45 degrees, suctioning oropharyngeal and tracheobronchial secretions, and recording pre-extubation monitoring on the corresponding sheet. Then, operator A left the site and was replaced by operators $\mathrm{B}$ and $\mathrm{C}$, who opened the assigned envelope. The extubation procedure and postextubation oxygen delivery were performed by operator B. Regardless of the extubation technique used, the subjects received supplemental oxygen through a nasal cannula placed before ETT removal to maintain the same flow level used in the spontaneous breathing trial. Operator $\mathrm{C}$ was involved only when the extubation procedure corresponded to the traditional technique. Before extubation and regardless of the procedure assigned, the alarms were silenced and the ventilator parameters were set for PSV mode (inspiratory pressure of $15 \mathrm{~cm} \mathrm{H}_{2} \mathrm{O}$ and PEEP of $10 \mathrm{~cm} \mathrm{H}_{2} \mathrm{O}$ ) by operator B, so that operator A could not hear the maneuver or read the ventilator parameters.

Once extubation was performed, assessment of outcome measures recorded within $60 \mathrm{~min}$ after extubation was done by operator $\mathrm{A}$, who was blinded to the procedure. Daily monitoring and recording of outcomes within $72 \mathrm{~h}$ of extubation was carried out retrospectively by this same operator. The decision to perform extubation and to use NIV to prevent extubation failure, as well as subsequent follow-up and treatment, was undertaken by the respiratory therapist on call, who was blinded to randomization assignment. If preventive NIV had previously been decided, then it was initiated $15 \mathrm{~min}$ after extubation so as not to affect monitoring of outcome measures.

\section{Outcome Measures}

\section{Primary Outcome Measure}

Postextubation overall complications: Clinical evidence of at least one of the following:
- Either at 1, 3, 5, or 15 min after extubation:

- Desaturation $3,6,19: \mathrm{S}_{\mathrm{pO}_{2}}<90 \%$ or a 4-point decrease compared with pre-extubation $\mathrm{S}_{\mathrm{pO}_{2}} \cdot{ }^{5}$ A lower value maintained for at least $10 \mathrm{~s}$ was recorded.

- Hypertension ${ }^{3,4,19}$ : systolic blood pressure $>180 \mathrm{~mm}$ $\mathrm{Hg}$ or increased by $>20 \%$ from pre-extubation. ${ }^{1}$

- Tachycardia ${ }^{3,4,19}$ : heart rate $>140$ beats/min or increased by $>20 \%$ from pre-extubation. ${ }^{1}$

- Tachypnea $^{20}$ : breathing frequency $>35$ breaths/min or increased by $>50 \%$ from pre-extubation. ${ }^{1}$

- Poor respiratory mechanics ${ }^{20}$ : the presence of thoracoabdominal asynchrony or inspiratory and/or expiratory effort, as observed by the evaluator.

- Within 60 min after extubation:

- Upper airway obstruction: the presence of laryngeal stridor, audible with or without a stethoscope. ${ }^{4,21}$

- Postobstructive pulmonary edema: defined as desaturation and pink frothy sputum after upper airway obstruction. ${ }^{2,21}$

- Vomiting. ${ }^{3,5}$

- Bronchospasm ${ }^{5,21}$ : audible with or without a stethoscope. Bronchospasm was considered a complication only in subjects not presenting the event during the T-piece test performed before orotracheal extubation.

- Severe Cough ${ }^{3,5}$ : defined as more than one episode of sustained cough $(>5 \mathrm{~s}) .^{22}$

\section{Secondary Outcome Measures}

- Postextubation major complication: clinical evidence of at least one of the following: upper airway obstruction, desaturation, vomiting.

- Postextubation minor complication: clinical evidence of at least one of the following: bronchospasm, severe cough, hypertension, tachycardia, tachypnea, poor respiratory mechanics.

- Postextubation pneumonia: the presence of fever, leukocytosis $\left(>10,000 / \mathrm{mm}^{3}\right)$, purulent secretions, and new pulmonary infiltrate on chest radiography within $72 \mathrm{~h}$ after extubation. ${ }^{23}$

- Extubation failure: use of NIV to treat the failure or need of reintubation within $72 \mathrm{~h}$ after extubation. ${ }^{1,24}$

- Reintubation: Within $72 \mathrm{~h}$ after extubation. ${ }^{1,7}$

\section{Other Outcome Measures}

The frequency of complications, days of invasive mechanical ventilation, use of postextubation preventive NIV, and length of critical care unit and hospital stay were recorded. 


\section{Sample Size}

Before initiating the study, a pilot trial with 20 subjects was performed. Based on a complication rate of $83 \%$, the sample size was determined for a power of $80 \%$. With assuming that positive pressure is not worse than the traditional technique, 198 subjects (99 per group) were required for the upper limit of a unilateral $97.5 \%$ CI to exclude a difference of $\geq 15 \%$ in favor of the traditional technique. Based on an interim analysis performed 6 months after the study was initiated, a complication rate of $78 \%$ was found. Thus, the required sample size was adjusted to 240 subjects (120 per group).

\section{Randomization}

Randomization sequence was generated by using the web site www.randomization.com (Accessed on November 20, 2015). The subjects were randomized in a 1:1 ratio to positive pressure or traditional technique. Closed, opaque, numbered envelopes were used to conceal group allocation. Randomization sequence and concealment were conducted by a person not involved in the study.

\section{Blinding}

Due to the nature of the intervention, blinding of the subject and the operators responsible for extubation was not possible. The person in charge of data statistical analysis and the evaluator who monitored and recorded outcome measures were blinded to the allocated intervention.

\section{Statistical Analysis}

The primary analysis of outcomes was a per protocol analysis. In addition, an intention-to-treat analysis was performed to check the robustness of the results. For the intention-to-treat analysis, a "worst case scenario" was applied, allocating the event of interest to those subjects randomly assigned to positive-pressure group and not allocating the event to those in traditional group. Continuous variables with normal distribution were presented as mean $\pm \mathrm{SD}$. Non-normally distributed variables were presented as median with interquartile range (IQR). Symmetry of distribution was determined by the KolmogorovSmirnov test. Categorical variables were presented as absolute number and percentage. For univariate comparison of continuous variables between the groups, the Student $t$ test or the Mann-Whitney test was used as appropriate. For categorical variables, the chi-square test or the Fisher exact test was used as appropriate.

Noninferiority and, when appropriate, nonsuperiority sequential analyses were performed. For noninferiority and superiority comparisons, a 1 - or 2-sided alpha value of 0.05 was

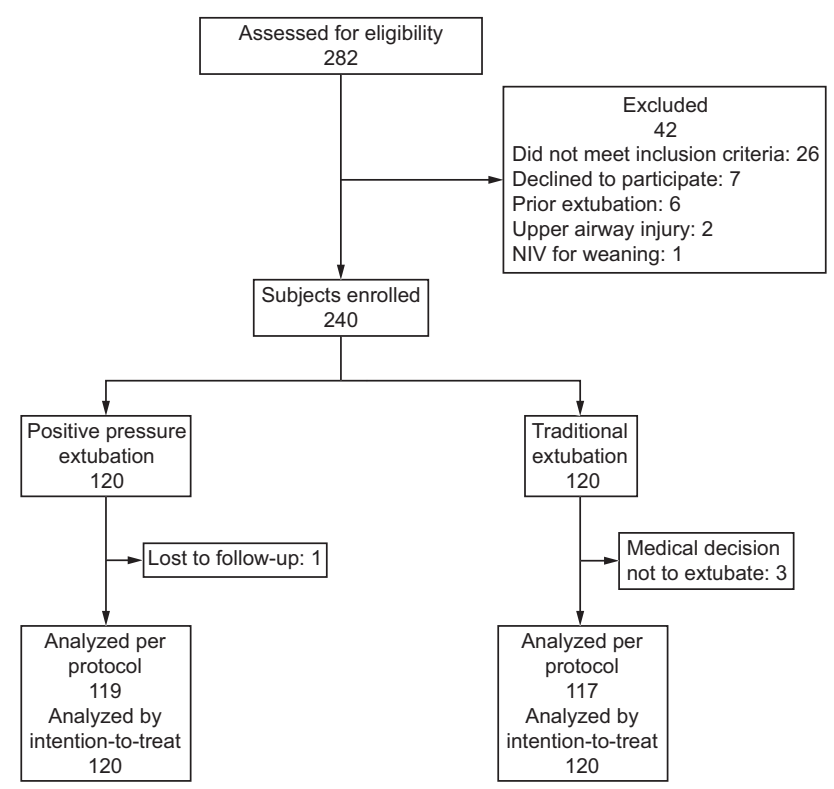

Fig. 1. Flow chart. Note, all 240 subjects were analyzed separately as intention to treat. NIV $=$ noninvasive ventilation.

used, respectively. The positive-pressure technique was considered noninferior to the traditional technique, if the upper limit of a 1-sided $97.5 \% \mathrm{CI}$ for the absolute risk difference between both techniques did not exceed a relative margin of $15 \%$ in favor of the traditional group, both in the per protocol and in the intention-to-treat analyses. For this purpose, a $\mathrm{Z}$ test for the difference in proportions was performed. A $P$ value of $<.05$ was considered significant. A post hoc sensitivity analysis was done to identify independent factors related to major complications while controlling for confounding variables. For this purpose, we constructed a conditional backwards stepwise multivariable logistic regression model, including the prespecified variables: age, sex, high risk of extubation failure, and those variables with $P<.15$ in the univariate analysis. The discrimination of the multivariate model was assessed by using the area under the receiver operating characteristic curve and the Hosmer-Lemeshow goodness-of-fit test. All statistical analyses were performed by using SPSS 24.0 (SPSS, Chicago, Illinois) and Minitab 18.0 software (Minitab, State College, Pennsylvania).

\section{Results}

A total of 282 subjects in critical care areas at the Hospital Donación Francisco Santojanni were recruited from January 2017 to March 2018. Four subjects were eliminated: 1 from the positive-pressure group and 3 from the traditional group. Screening, enrollment, and randomization of the participants is shown in Figure 1. No statistically significant differences were found in baseline characteristics and pre-extubation parameters between the groups (Tables 1 and 2). 
Positive Pressure Extubation Technique

Table 1. Demographic and Clinical Characteristics

\begin{tabular}{|c|c|c|c|}
\hline Variable & $\begin{array}{l}\text { Positive-Pressure } \\
\text { Extubation } \\
\text { Technique }\end{array}$ & $\begin{array}{l}\text { Traditional } \\
\text { Extubation } \\
\text { Technique }\end{array}$ & $P$ \\
\hline Subjects, $n$ & 119 & 117 & \\
\hline Age, median (IQR) y & $55(40-70)$ & $58(37-72)$ & .84 \\
\hline Females, $n(\%)$ & $50(42)$ & $38(32.5)$ & .13 \\
\hline APACHE II, median (IQR) score & $15(10-21)$ & $15(10-21)$ & .71 \\
\hline \multicolumn{4}{|l|}{ Comorbidities, $n(\%)$} \\
\hline COPD & $12(10.1)$ & $10(8.5)$ & .68 \\
\hline Smoking & $18(15.1)$ & $20(17.1)$ & .68 \\
\hline Illicit drugs & $7(5.9)$ & $1(9.4)$ & .31 \\
\hline Hypertension & $40(33.6)$ & $41(35)$ & .82 \\
\hline Alcoholism & $8(57.1)$ & $6(42.9)$ & .56 \\
\hline Diabetes & $20(16.8)$ & $23(19.7)$ & .57 \\
\hline Other & $42(35.9)$ & $32(26.9)$ & .14 \\
\hline $\begin{array}{l}\text { Subjects at high risk for } \\
\quad \text { extubation failure, } n(\%)\end{array}$ & $71(59.7)$ & $66(56.4)$ & .61 \\
\hline \multicolumn{4}{|l|}{$\begin{array}{l}\text { Risk factors for extubation } \\
\text { failure, } n(\%)\end{array}$} \\
\hline Anemia & $8(6.7)$ & $11(9.4)$ & .45 \\
\hline Obstructive sleep apnea & $1(0.8)$ & $1(0.9)$ & .75 \\
\hline Age $>65$ y & $44(37)$ & $43(36.8)$ & .97 \\
\hline Head and neck pathology & $3(2.5)$ & $4(3.4)$ & .49 \\
\hline Heart disease & $36(30.3)$ & $35(29.9)$ & .95 \\
\hline Lung disease & $9(7.6)$ & $9(7.7)$ & .97 \\
\hline Obesity & $20(16.8)$ & $16(13.7)$ & .50 \\
\hline \multicolumn{4}{|l|}{ Critical care unit, $n(\%)$} \\
\hline ICU & $53(44.5)$ & $50(42.7)$ & .78 \\
\hline Emergency department & $41(34.5)$ & $43(36.8)$ & .71 \\
\hline Coronary unit & $25(21)$ & $24(20.5)$ & .92 \\
\hline \multicolumn{4}{|l|}{$\begin{array}{l}\text { Reason for mechanical } \\
\text { ventilation, } n(\%)\end{array}$} \\
\hline Acute respiratory failure & 84 (70.6) & $75(64.1)$ & .29 \\
\hline Postoperative & $39(46.4)$ & $33(43.4)$ & .45 \\
\hline Trauma & $4(4.8)$ & $6(7.9)$ & .54 \\
\hline Congestive heart failure & $10(11.9)$ & $7(9.2)$ & .47 \\
\hline Pneumonia & $15(17.9)$ & $7(9.2)$ & .08 \\
\hline Sepsis & $7(8.3)$ & $11(14.5)$ & .31 \\
\hline ARDS & $1(1.2)$ & $1(1.3)$ & $>.99$ \\
\hline Aspiration & $2(2.4)$ & $0(0)$ & .24 \\
\hline Cardiac arrest & $2(2.4)$ & $1(1.3)$ & $>.99$ \\
\hline Other & $6(7.1)$ & $7(10.5)$ & .75 \\
\hline Chronic respiratory failure & $4(3.4)$ & $2(1.7)$ & .68 \\
\hline COPD & $3(75)$ & $1(50)$ & .62 \\
\hline Asthma & $1(25)$ & $1(50)$ & $>.99$ \\
\hline Coma & $30(25.2)$ & $39(33.3)$ & .17 \\
\hline Stroke & $11(36.7)$ & $11(28.2)$ & .97 \\
\hline Traumatic brain injury & $8(26.7)$ & $13(33.3)$ & .24 \\
\hline Metabolic & $2(6.7)$ & $2(5.1)$ & $>.99$ \\
\hline Poisoning & $2(6.7)$ & $4(10.3)$ & .44 \\
\hline Other & $7(23.3)$ & $9(23.1)$ & .58 \\
\hline Neuromuscular & $1(0.8)$ & $1(0.8)$ & $>.99$ \\
\hline $\begin{array}{l}\text { Mechanical ventilation before } \\
\text { extubation, median (IQR) d }\end{array}$ & $4(1-8)$ & $3(1-6.5)$ & .38 \\
\hline
\end{tabular}

$\overline{\mathrm{IQR}=\text { interquartile range }}$

APACHE $=$ Acute Physiology and Chronic Health Evaluation
Table 2. Pre-Extubation Characteristics

\begin{tabular}{|c|c|c|c|}
\hline Variable & $\begin{array}{c}\text { Positive-Pressure } \\
\text { Extubation } \\
\text { Technique }\end{array}$ & $\begin{array}{l}\text { Traditional } \\
\text { Extubation } \\
\text { Technique }\end{array}$ & $P$ \\
\hline Subjects, $n$ & 119 & 117 & \\
\hline Tidal volume, $\mathrm{mL} / \mathrm{kg}$ & $7.3(6.7-8)$ & $7.4(7-8)$ & .92 \\
\hline $\begin{array}{c}\text { Endotracheal tube } \\
\text { diameter, mm }\end{array}$ & $8(7.5-8)$ & $8(7.5-8)$ & .14 \\
\hline $\mathrm{P}_{\mathrm{aO}_{2}} / \mathrm{F}_{\mathrm{IO}_{2}}$ & $358(286-400)$ & $357(258-400)$ & .70 \\
\hline $\mathrm{F}_{\mathrm{IO}_{2}}, \%$ & $30(30-40)$ & $30(30-38.7)$ & .25 \\
\hline PEEP, $\mathrm{cm} \mathrm{H}_{2} \mathrm{O}$ & $5(5-8)$ & $5(5-6)$ & .20 \\
\hline $\begin{array}{l}\text { Frequency, } \\
\quad \text { breaths/min }\end{array}$ & $20(16-24)$ & $20(16-24)$ & .82 \\
\hline $\begin{array}{l}\text { Heart rate, mean } \pm \text { SD } \\
\text { beats } / \text { min }\end{array}$ & $92.7 \pm 16.6$ & $90.8 \pm 14.7$ & .36 \\
\hline $\mathrm{S}_{\mathrm{pO}_{2}}, \%$ & $98(96-99)$ & $98(96-99)$ & .82 \\
\hline $\begin{array}{l}\text { Systolic blood pressure, } \\
\text { mm Hg }\end{array}$ & $137(124-153)$ & $135(119-150)$ & .28 \\
\hline $\begin{array}{l}\text { Diastolic blood pressure, } \\
\text { mm Hg }\end{array}$ & $76(67-84)$ & $76(68-88)$ & .95 \\
\hline
\end{tabular}

\section{Primary Results}

A total of 168 subjects $(71.2 \%)$ presented with at least one complication. The incidence of overall complications in the positive-pressure group was $65.5 \%$ (78/119) compared with $76.9 \%(90 / 117)$ in the traditional group, with statistical significance for noninferiority both in the per protocol analysis (absolute risk difference $-11.4 \%$, $95 \% \mathrm{CI}-22.8$ to $0 \%, P<.001)$ and in the intention-totreat analysis (absolute risk difference $-9.2 \%$, $95 \%$ CI -20.6 to $2.3 \%, P<.001)$. No statistically significant differences were found in overall complications between the groups for the superiority comparison $(P=.05$ and $P=.12$ for per protocol and intention-to-treat analyses, respectively) (Fig. 2 and Table 3).

\section{Secondary Results}

In the positive-pressure group, the incidence rates for major complications, minor complications, pneumonia, extubation failure, and reintubation were lower than those observed in the traditional group, with statistical significance for noninferiority both in the per protocol and in the intention-to-treat analyses $(P<.001)$ (Fig. 2 and Table 3). The rate of major complications was lower in the positivepressure group than in the traditional group, with statistical significance for the superiority comparison, both in the per protocol analysis (absolute risk difference $-13.2 \%$, 95\% CI -24.9 to $-1.5 \%$ ]; $P=.03$ ) and the intention-to-treat analysis (absolute risk difference $-11.7 \%, 95 \% \mathrm{CI}-23.2$ to $-0.1 \%$ ]; $P=.049$ ) (Fig. 3 and Table 3). In the post hoc 


\section{Positive Pressure Extubation Technique}

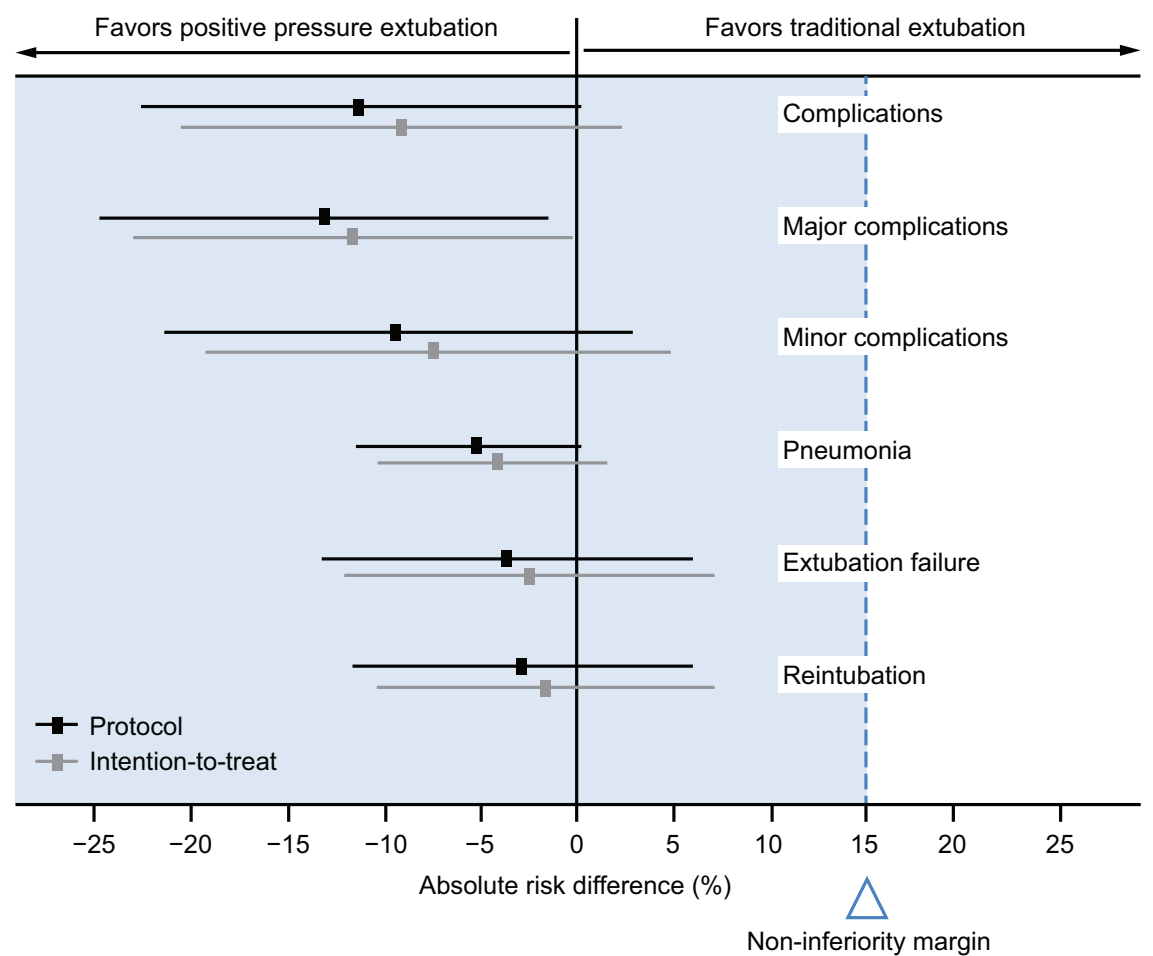

Fig. 2. Primary and secondary outcomes. Bar lines represent $95 \% \mathrm{Cls}$. The shaded area shows the noninferiority region, delimited by a noninferiority margin (dashed line) set at 15\%, which means that the positive-pressure extubation is noninferior if the upper limit of the $\mathrm{Cl}$ lies to the left of the dashed line, which indicates the noninferiority margin.

Table 3. Analysis of Primary and Secondary Outcomes

\begin{tabular}{|c|c|c|c|c|}
\hline Analysis & $\begin{array}{c}\text { Positive-Pressure } \\
\text { Extubation Technique, } n(\%)\end{array}$ & $\begin{array}{l}\text { Traditional Extubation } \\
\text { Technique, } n(\%)\end{array}$ & $\begin{array}{c}\text { Absolute Risk } \\
\text { Difference }(95 \% \mathrm{CI})\end{array}$ & $P^{*}$ \\
\hline \multicolumn{5}{|l|}{ Per protocol analysis } \\
\hline Subjects & 119 & 117 & & \\
\hline Complications & $78(65.5)$ & $90(76.9)$ & $-11.4(-22.8$ to 0$)$ & $<.001 .05 \dagger$ \\
\hline Major complications & $29(24.4)$ & $44(37.6)$ & $-13.2(-24.9$ to -1.5$)$ & $<.001 .03 \dagger$ \\
\hline Minor complications & $69(58)$ & $79(67.5)$ & $-9.5(-21.8$ to 2.7$)$ & $<.001 .12 \dagger$ \\
\hline Pneumonia & $2(1.7)$ & $8(6.8)$ & $-5.1(-10.3$ to 0$)$ & $<.001 .058 \dagger+$ \\
\hline Extubation failure & $18(15.1)$ & $22(18.8)$ & $-3.7(-13.2$ to 5.9$)$ & $<.001 .45 \dagger$ \\
\hline Reintubation & $14(11.8)$ & $17(14.5)$ & $-2.8(-11.4$ to 5.8$)$ & $<.001 .53 \dagger$ \\
\hline \multicolumn{5}{|l|}{ Intention-to-treat analysis } \\
\hline Subjects & 120 & 120 & & \\
\hline Complications & $79(65.8)$ & $90(75)$ & $-9.2(-20.6$ to 2.3$)$ & $<.001 .12 \dagger$ \\
\hline Major complications & $30(25)$ & $44(36.7)$ & -11.7 ( -23.2 to -0.1 & $<.001 .049 \dagger$ \\
\hline Minor complications & $70(58.3)$ & $79(65.8)$ & $-7.5(-19.7$ to 4.7$)$ & $<.001 .23 \dagger$ \\
\hline Pneumonia, & $3(2.5)$ & $8(6.7)$ & $-4.2(-9.4$ to 1.1$)$ & $<.001 .22 \dagger \dagger$ \\
\hline Extubation failure & $19(15.8)$ & $22(18.3)$ & $-2.5(-12$ to 7$)$ & $<.001 .61 \dagger$ \\
\hline Reintubation & $15(12.5)$ & $17(14.2)$ & $-1.7(-10.3$ to 6.9$)$ & $<.001 .70 \dagger$ \\
\hline $\begin{array}{l}* \text { All } P \text { values are } 1 \text {-sided and } \\
\dagger \text { Two-sided } P \text { value for super } \\
\ddagger P \text { value for the Fisher exact }\end{array}$ & ity, unless otherwise stated. & & & \\
\hline
\end{tabular}




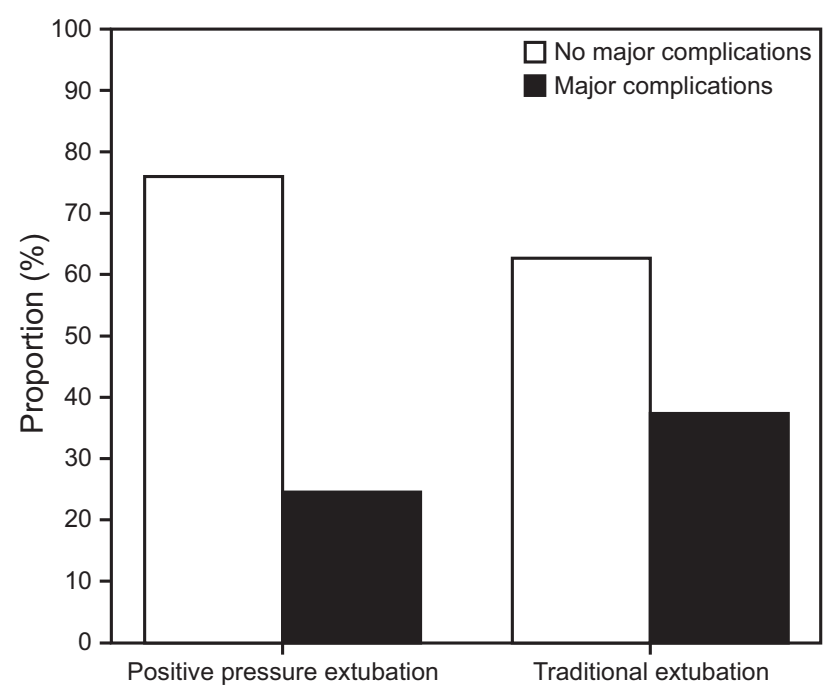

Fig. 3. Major complications per group in the per protocol analysis $(n=236)$.

Table 4. Logistic Regression for Major Complications

\begin{tabular}{lcc}
\hline \hline \multicolumn{1}{c}{ Variable } & $\begin{array}{c}\text { Odds Ratio } \\
(95 \% \text { CI })\end{array}$ & $P$ \\
\hline COPD & $2.26(0.90-5.69)$ & .08 \\
$\begin{array}{l}\text { Mechanical ventilation } \\
\text { before extubation } \geq 4 \mathrm{~d}\end{array}$ & $1.71(0.94-3.10)$ & .08 \\
APACHE II score $\geq 15$ & $1.68(0.93-3.03)$ & .09 \\
$\begin{array}{l}\text { Positive-pressure extubation } \\
\text { technique }\end{array}$ & $0.47(0.26-0.85)$ & .01 \\
\hline$P$ value of 0.82 for Hosmer-Lemeshow goodness of fit test. & \\
Area under the receiver operating characterístic curve was $0.66,95 \%$ CI 0.59 to 0.74. & \\
APACHE $=$ Acute Physiology and Chronic Health Evaluation & \\
\hline
\end{tabular}

sensitivity analysis, the multivariate regression model showed that positive pressure was an independent factor associated with a lower incidence of major complications (odds ratio $0.47,95 \%$ CI $0.26-0.85 ; P=.01$ ) (Table 4). No statistically significant differences were found in the superiority comparison for minor complications, pneumonia, extubation failure, and reintubation (Fig. 2 and Table 3).

\section{Other Results}

Of 326 total complications, the most common was hypertension (20.6\%), followed by desaturation (17.5\%), and tachycardia (17.2\%). No subjects developed postextubation pulmonary edema (Fig. 4). In 9 subjects $(7.6 \%)$ in the positive-pressure group and 7 of the traditional group (6\%), NIV was used to prevent extubation failure $(P=.63)$. The median (IQR) duration of invasive mechanical ventilation before extubation was 4
(1-9) and 3 (1-8) days in the positive-pressure and traditional groups, respectively $(P=.60)$. The median (IQR) length of critical care unit stay was $10(4-15)$ and $9(4-15)$ days in the positive-pressure and the traditional groups, respectively $(P=.96)$. The median (IQR) length of the hospital stay was $21(12-37.5)$ and 25 (12-44.5) days in the positive-pressure and traditional groups, respectively $(P=.44)$.

\section{Discussion}

The main result of this study was that positive pressure was shown to be a safe extubation technique and was noninferior to the traditional technique. One of the study findings was that all outcome estimations favored the positive-pressure technique, which was significantly superior in terms of incidence of major complications. Although the clinical magnitude of the difference observed was uncertain, the risk of a major complication was reduced by $>13 \%$ with the use of positive pressure (number needed to treat, 7.6), which means that, for every 7 subjects extubated with positive pressure, one major complication could be avoided. The multivariate sensitivity analysis validated the robustness of our results.

This finding and the trend toward a lower incidence of minor complications in the positive-pressure group could be explained by the lower rate of airway obstruction and severe cough, respectively. Positive pressure extubation involves involves less leakage of secretions pooled in the subglottic space to the distal airway and does not require endotracheal suction during ETT removal, which leads to less airway stimulation. ${ }^{9,11}$ Impaired oxygenation is one of the most commonly reported extubation complications, ${ }^{3}$ and it has been associated with reduction of lung volume and oxygen stores during endotracheal suction. This is the reason why it has been the primary outcome measure in studies that compared traditional and positivepressure extubation techniques. ${ }^{6,12,25}$ The findings were conflicting; whereas our results showed similar between-group values, which coincided with what was reported by L'Hermite et al, ${ }^{25}$ some investigators described a higher incidence of this complication with negative pressure extubation. ${ }^{6,12}$

The incidence of postextubation pneumonia was lower in the positive-pressure group, but no significant differences were found between the groups. Positive pressure could reduce the leakage of subglottic colonized secretions into the distal airway. ${ }^{9,11}$ However, the development of pneumonia depends on multiple factors. ${ }^{26}$ The rate of extubation failure and reintubation found in both groups is consistent with that reported in the literature. ${ }^{23,24}$ Although a lower frequency of complications may lead to lower rates of extubation failure and subsequent reintubation, it is important to consider that such complications are not only related to aspects inherent to ETT removal but also to 


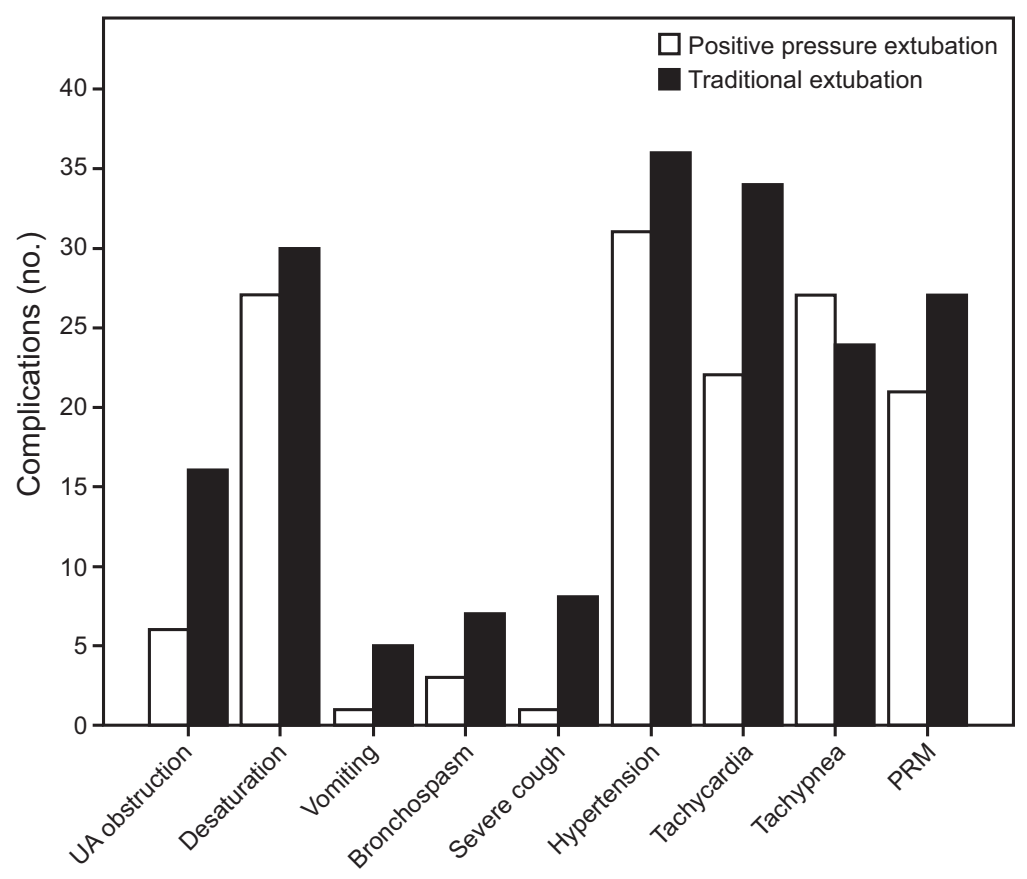

Fig. 4. Frequency of complications. $U A=$ upper airway, $\mathrm{PRM}=$ poor respiratory mechanics.

subject factors associated with spontaneous ventilation through their natural airway.

A total of 326 complications were recorded, with the most common being hypertension. This could be a result of extubation, which induces an adrenergic discharge. Tachycardia, the third most common complication, would support this theory. ${ }^{27}$ Likewise, it has been shown that severe cough could increase hypertension. ${ }^{28}$ It should be noted that, because the complications mentioned above are not isolated events, one of these complications can impact the others, which can affect the result interpretation. ${ }^{13} \mathrm{~A}$ strength of this study was that the heterogeneous population studied, which consisted of clinical and surgery subjects who were critically ill, makes it possible to extrapolate and generalize our results to various critical intensive care populations.

First, one of the study limitations was that, due to the lack of available evidence, the noninferiority margin was defined based on expert opinion. Although it may be considered a wide margin, all outcomes favored the positivepressure technique, and no upper margin of the 95\% CIs favored the traditional technique by $>6 \%$. Second, we emphasized the lack of diagnostic accuracy for postextubation pneumonia because of the impossibility of having microbiological criteria from invasive and noninvasive sampling. However, it was determined based on the presence of objective clinical, laboratory, and radiologic established criteria, which guide usual clinical practice in our institution. Third, and from a statistical perspective, a post hoc analysis showed that the actual study power to detect the reduction seen in the incidence of major complications was 0.5 . Nevertheless, and as a result of the statistically significant differences found with this study sample size, we considered that a larger sample size would further support our results.

\section{Clinical and Research Implications}

Positive pressure is noninferior to traditional in terms of safety because it does not result in higher incidence of overall, major or minor complications, pneumonia, or extubation failure, and reintubation rates. In addition, the positive-pressure technique was to be superior, which revealed a lower incidence of major complications. For every 7 subjects extubated by using positive pressure, one major complication would be avoided. Positive pressure does not require more medical devices or equipment than those usually needed for the traditional technique. To our knowledge, this was the first study that compared both extubation techniques with regard to potential complications in subjects who were critically ill. Our results encourage further studies to confirm the superiority of positive pressure.

\section{Conclusions}

This study proved that positive pressure was noninferior to the traditional technique in terms of safety because it does not result in a higher incidence of overall, major or minor complications, pneumonia, or extubation failure and 


\section{Positive Pressure Extubation Technique}

reintubation rates. In addition, positive pressure was shown to be superior in terms of a lower incidence of major complications. Thus, we consider that it should be used in daily clinical practice within critical care units.

\section{REFERENCES}

1. Boles JM, Bion J, Connors A, Herridge M, Marsh B, Melot C, et al. Weaning from mechanical ventilation. Eur Respir J 2007;29(5): 10331056.

2. Artime CA, Hagberg CA. Tracheal extubation. Respir Care 2014; 59(6):991-1002; discussion 1002-1005.

3. Rassam S, Sandbythomas M, Vaughan RS, Hall JE. Airway management before, during and after extubation: a survey of practice in the United Kingdom and Ireland. Anaesthesia 2005;60(10):995-1001.

4. Difficult Airway Society Extubation Guidelines Group. Popat M, Mitchell V, Dravid R, Patel A, Swampillai C, Higgs A. Difficult Airway Society Guidelines for the management of tracheal extubation. Anaesthesia 2012;67(3):318-340.

5. Asai T, Koga G, Vaughan RS. Respiratory complications associated with tracheal intubation and extubation. Br J Anaesth 1998;80(6): 767-775.

6. Guglielminotti J, Constant I, Murat I. Evaluation of routine tracheal extubation in children: inflating or suctioning technique? Br J Anaesth 1998;81(5):692-695.

7. Epstein SK. Decision to extubate. Intensive Care Med 2002;28(5): 535-546.

8. Scales K, Pilsworth J. A practical guide to extubation. Nurs Stand 2007;22(2):44-48

9. Hodd J, Doyle A, Carter J, Albarran J, Young P. Increasing positive end expiratory pressure at extubation reduces subglottic secretion aspiration in a bench-top model. Nurs Crit Care 2010;15(5):257-261.

10. Dave MH, Frotzler A, Madjdpour C, Koepfer N, Weiss M. Massive aspiration past the tracheal tube cuff caused by closed suction system. J Intensive Care Med 2011;26(5):326-329.

11. Andreu MF, Salvati IG, Donnianni MC, Ibañez B, Cotignola M, Bezzi M. Effect of applying positive pressure with or without endotracheal suctioning during extubation: a laboratory study. Respir Care 2014;59(12):1905-1911.

12. Yousefshahi F, Barkhordari K, Movafegh A, Tavakoli V, Paknejad $\mathrm{O}$, Bina $\mathrm{P}$, et al. A new method for extubation: comparison between conventional and new methods. J Tehran Heart Cent 2012;7(3):121127.

13. Piaggio G, Elbourne DR, Pocock SJ, Evans SJ, Altman DG; CONSORT group. Reporting of noninferiority and equivalence randomized trials: extension of the CONSORT 2010 statement. JAMA 2012;308(24):2594-2604

14. Moher D, Hopewell S, Schulz KF, Montori V, Gøtzsche PC, Devereaux PJ, et al.; Consolidated Standards of Reporting Trials Group. CONSORT 2010 Explanation and Elaboration: updated guidelines for reporting parallel group randomized trials. J Clin Epidemiol 2010; 63(8):e1-37.

15. Esteban A, Alia I, Tobin MJ, Gil A, Gordo F, Vallverdu I, et al Effect of spontaneous breathing trial duration on outcome of attempts to discontinue mechanical ventilation. Spanish Lung Failure Collaborative Group. Am J Respir Crit Care Med 1999;159(2):512518.

16. Vidotto MC, Sogame LC, Gazzotti MR, Prandini MN, Jardim JR. Analysis of risk factors for extubation failure in subjects submitted to non-emergency elective intracranial surgery. Respir Care 2012; 57(12):2059-2066

17. Khamiees M, Raju P, DeGirolamo A, Amoateng-Adjepong Y, Manthous CA. Predictors of extubation outcome in patients who have successfully completed a spontaneous breathing trial. Chest 2001; 120(4):1262-1270

18. Thille AW, Boissier F, Ben Ghezala H, Razazi K, Mekontso-Dessap A, Brun-Buisson C. Risk factors for and prediction by caregivers of extubation failure in ICU patients: a prospective study. Crit Care Med 2015;43(3):613-620.

19. Richardson PB, Krishnan S, Janakiraman C, Wilkes AR, Hodzovic I. Extubation after anaesthesia: a randomized comparison of three techniques. Acta Clin Croat 2012;51(3):529-536.

20. Epstein SK. Endotracheal extubation. Respir Care Clin N Am 2000; 6(2):321-360,vi.

21. American Association for Respir Care - AARC. AARC Clinical Practice Guidelines. Removal of the Endotracheal Tube-2007 Revision and Update. Respir Care 2007;52(1):81-93. Available at http:// rc.rcjournal.com/content/respcare/52/1/81.full.pdf.

22. Minogue SC, Ralph J, Lampa MJ. Laryngotracheal topicalization with lidocaine before intubation decreases the incidence of coughing on emergence from general anesthesia. Anesth Analg 2004;99(4): 1253-1257, table of contents.

23. Thille AW, Harrois A, Schortgen F, Brun-Buisson C, Brochard L. Outcomes of extubation failure in medical intensive care unit patients. Crit Care Med 2011;39(12):2612-2618.

24. Epstein SK, Ciubotaru RL, Wong JB. Effect of failed extubation on the outcome of mechanical ventilation. Chest 1997;112(1):186-192.

25. L'Hermite J, Wira O, Castelli C, de la Coussaye JE, Ripart J, Cuvillon $\mathrm{P}$. Tracheal extubation with suction vs positive pressure during emergence from general anaesthesia in adults: a randomized controlled trial. Anaesth Crit Care Pain Med 2018;37(2):147-153.

26. American Thoracic Society. Infectious Diseases Society of America. Guidelines for the management of adults with hospital-acquired, ventilator-associated, and healthcare-associated pneumonia. Am J Respir Crit Care Med 2005;171(4):388-416.

27. Miller KA, Harkin CP, Bailey PL. Postoperative tracheal extubation. Anesth Analg 1995;80(1):149-172.

28. Kern MJ, Gudipati C, Tatineni S, Aguirre F, Serota H, Deligonul U. Effect of abruptly increased intrathoracic pressure on coronary blood flow velocity in patients. Am Heart J 1990;119(4):863-870.

This article is approved for Continuing Respiratory Care Education credit. For information and to obtain your CRCE

(free to AARC members) visit www.rcjournal.com

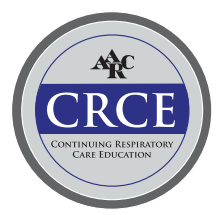

